\title{
Strategic placement of epidural catheter
}

\author{
Jae-Hang Shim \\ Department of Anesthesiology and Pain Medicine, Hanyang University Hospital, Guri, Korea
}

Epidural catheter insertion is useful for long term postoperative pain control or prolonged regional anesthesia for an operation, moreover, pain therapy for back pain or radiating pain $[1,2]$. For expected effect of epidural block, correct placement of epidural catheter is most important. However, the primary failure rate of epidural space catheterization can exceed $10 \%$ [3].

There is no objective, reliable, real time method to determine the correct catheter placement in the epidural space. Epidural test dose injection technique (2-3 ml lidocaine $1.5 \%)$ is usually used for verification of epidural catheter. But there is false positive and negative associated with the standard test dose injection [4]. Epidurography is also a useful modality for confirming location of the catheter. However, it is not a simple method because c-arm fluoroscope and contrast media are needed. Asato et al. [5] showed that the most frequent cause of unilateral epidural blockade was the misplacement of the catheter into the anterior epidural space. Tsui et al. [6] mentioned the use of low current epidural stimulation to confirm the epidural catheter placement for the first time. Epidural stimulation test (EST) is more accurate than standard test dose technique, and is deemed to be simpler than epidurography. The EST criteria define correct localization of the catheter tip in the epidural space by elicited motor response with a current between $1-10 \mathrm{~mA}$ at a frequency of $1 \mathrm{~Hz}$ with a pulse width of $0.2 \mathrm{~ms}[6,7]$. There are truncal or limb movements when epidural catheter is correctly located. Appropriate muscle contractions consist of rhythmic hip adductors, iliopsoas, gluteus muscles and hamstring group movement on lumbar EST. If bilateral motor response was exhibited at an unusually low current, subarachnoid or epiradicular placement of catheter is considered.
In this issue of the Korean Journal of Anesthesiology, Jung et al. [8] report on confirming the localization of an epiradicular catheter placement with different electrical pulse width. In the current study, instead of the threshold current, they compared the threshold charge. The number of charges (in nanocoulombs; nC) delivered is equal to the product of the current (in milliAmperes) and the pulse width (in milliseconds) [9]. Tsui et al. applied electric current between 1-10 mA with a pulse width of $0.2 \mathrm{~ms}$ for EST [6]. Pulse width of $0.2 \mathrm{~ms}$ is better than higher pulse width for EST because $0.2 \mathrm{~ms}$ is closer to the motor fiber's chronaxie. However, some nerve stimulator can't serve wide ranges of pulse width and current of electricity. Threshold charge might be a good parameter for determining correct catheter placement with variable kind of nerve stimulators.

They used a single port, metal coil-reinforced, 19-gauge epidural catheter, instead of the conventional epidural catheter, which was usually used. It has an advantage that does not need normal saline for electrical conduction during EST. As the effective conduction of electricity is essential for the success of EST, the type of catheter is important. TheraCath, which they chose, provided an effective electrostimulation of the epidural space and offered an alternative to the traditional method of using a catheter primed with normal saline [10].

In this study, the electrical stimulation test performance results must be interpreted cautiously due to the specific patients group enrolled. They evaluated the threshold current of the patients group with spinal pathology. Tsui et al. [6] mentioned motor response at a current $<1 \mathrm{~mA}$ revealed an intrathecal, subdural or epiradicular placement of epidural catheter. The lower cutoff $<1 \mathrm{~mA}$ should not be considered an absolute limit but always a warning sign for possible epiradicular, subdural

Corresponding author: Jae-Hang Shim, M.D., Ph.D., Department of Anesthesiology and Pain Medicine, Hanyang University Hospital, 249-1, Gyomun-dong, Guri 471-701, Korea. Tel: 82-31-560-2390, Fax: 82-31-563-1731, E-mail: jhshim@hanyang.ac.kr

(c) This is an open-access article distributed under the terms of the Creative Commons Attribution Non-Commercial License (http:// creativecommons.org/licenses/by-nc/3.0/), which permits unrestricted non-commercial use, distribution, and reproduction in any medium, provided the original work is properly cited. 
or subarachnoid position [11]. Epiradicular catheters were inserted for patients complaining of low extremity radiating pain due to spinal pathology. It is just a changing Tsui's way of thinking. They hypothesized that epiradicular current threshold of patients who have the degenerated nerve root or pathologic condition of spinal cord might be increased, compared with normal patients. Epiradicular threshold current (or charges) overlapped the epidural threshold current (or charges). The overlapping of the threshold current might be explained by adjacency of the catheter tip to the nerve root and chronic compression resulting in microvascular injury. The threshold current of epiradicular catheter could be useful for verifying spinal pathology, such as inflammation for patients undergoing back pain or radiating pain if more data is accumulated.

EST is more accurate and simple real time method for confirming the localization of an epiradicular catheter placement. Moreover, it would make diagnosis of spinal pathology possible. Nonetheless, further studies are required before implementing its routine use in clinical settings.

\section{References}

1. Dolin SJ, Cashman JN, Bland JM. Effectiveness of acute postoperative pain management: I. Evidence from published data. Br J Anaesth 2002; 89: 409-23.
2. Block BM, Liu SS, Rowlingson AJ, Cowan AR, Cowan JA Jr, Wu CL. Efficacy of postoperative epidural analgesia: a meta-analysis. JAMA 2003; 290: 2455-63.

3. Rigg JR, Jamrozik K, Myles PS, Silbert BS, Peyton PJ, Parsons RW, et al. Epidural anaesthesia and analgesia and outcome of major surgery: a randomised trial. Lancet 2002; 359: 1276-82.

4. Richardson MG, Lee AC, Wissler RN. High spinal anesthesia after epidural test dose administration in five obstetric patients. Reg Anesth 1996; 21: 119-23.

5. Asato F, Goto F. Radiographic findings of unilateral epidural block. Anesth Analg 1996; 83: 519-22.

6. Tsui BC, Gupta S, Finucane B. Confirmation of epidural catheter placement using nerve stimulation. Can J Anaesth 1998; 45: 640-4.

7. Tsui BC. Epidural stimulation test criteria. Anesth Analg 2006; 103: 775-6.

8. Jeong JS, Shim JC, Shim JH, Kim DW, Kang MS. Minimum current requirement for confirming the localization of an epiradicular catheter placement. Korean J Anesthesiol 2012; 63: 238-44.

9. Hadzic A, Vloka J, Hadzic N, Thys DM, Santos AC. Nerve stimulators used for peripheral nerve blocks vary in their electrical characteristics. Anesthesiology 2003; 98: 969-74.

10. Charghi R, Chan SY, Kardash KJ, Finlayson RJ, Tran De QH. Electrical stimulation of the epidural space using a catheter with a removable stylet. Reg Anesth Pain Med 2007; 32: 152-6.

11. Lena P, Martin R. Subdural placement of an epidural catheter detected by nerve stimulation. Can J Anaesth 2005; 52: 618-21. 